\title{
Controllable Motions of Compressible Simple Materials of Various Types
}

\author{
By
}

\author{
A. S. Wineman, Ann Arbor, Mich.
}

(Received December 11, 196\%)

\section{Summary - Zusammenfassung}

Controllable Motions of Compressible Simple Materials of Various Types. It is shown that a motion is possible in every compressible homogeneous isotropic simple solid having a certain range of memory, in the presence of a constant body force field, if and only if it is homogeneous and uniformly accelerated after a certain time. In the class of solids having perfect memory, the only motion of this kind which can be smoothly initiated in a body at rest is a rigid one. For the class of solids having finite memory of duration $T$ and initially at rest, the motions of the type considered need be homogeneous and uniformly accelerated only after time $T$. For those solids having fading memory, the motion must reduce to a fixed homogeneous deformation. Similar results are developed for simple fluids and anisotropic simple solids.

Steuerbare Bewegungen versehiedener kompressibler einfacher Stoffe. Es wird gezeigt, daß in jedem kompressiblen, homogenen und isotropen einfachen Festkörper mit einem gewissen Erinnerungsbereich und in Gegenwart eines konstanten Massenkraftfeldes eine Bewegung dann und nur dann möglich ist, wenn sie homogen und nach einer gewissen Zeit gleichmäßig beschleunigt ist. Bei Festkörpern mit vollkommener Erinnerung ist die einzige Bewegung dieser Art, die bei einem ruhenden Körper glatt eingeleitet werden kann, eine starre. Bei Festkörpern mit begrenzter Erinnerungsdauer $T$, die anfangs in Ruhe waren, müssen die Bewegungen der betrachteten Art homogen und nur nach der Zeit $T$ gleichmäßig beschleunigt sein. Für Festkörper mit schwindendem Gedächtnis reduziert sich die Bewegung auf eien feste homogene Verformung. Ähnliche Ergebnisse werden für einfache Flüssigkeiten und anisotrope einfache Festkörper entwickelt.

\section{Introduction}

A simple material, as defined by NoLL $[1]^{1}$, is one for which knowledge of its response to all homogeneous deformations is necessary and sufficient to determine the relation between stress and any deformation. It thus appears that in order to determine the response functional relating stress and deformation history, one need only carry out an experimental program in which a specimen is subjected to arbitrary homogeneous deformations. The possibility of carrying out such a program depends on

1 Numbers in square brackets refer to the List of References at the end of this paper. 
whether the material is incompressible or compressible. Coleman and TRUESDELL [2] have shown that only irrotational homogeneous motions need be considered for incompressible materials. For compressible materials, TRUESDELL and Noli $[3, \S 28]$ have shown that, in the presence of a constant body force field, the only homogeneous motions satisfying the equations of motion are those having constant acceleration. In order to determine the response functional for compressible materials therefore, it will be necessary to subject the material to nonhomogeneous deformations. In this case the experimentor is faced with the problem that, in general such motions depend on the form of the response functional he is trying to determine.

Suppose an experimenter has decided that a given specimen is a compressible homogeneous isotropic solid. With no other a priori prejudice as to the nature of the material, and with the above remarks in mind, the question now arise as to whether there are any motions which may be produced in every compressible homogeneous isotropic solid. Such a motion must then be possible in the particular specimen on hand. For any motion of this kind the interior state of deformation at each time $t$ will be the same for all such isotropic solids. Assuming a constant body force field only, these motions can be produced by the application of appropriate surface tractions alone. Knowing the details of the deformation completely, and having measured the surface tractions required, the experimenter can directly obtain information on the form of the response functional. We call such motions controllable. A more precise definition will be given in Section 3.

In the present paper we will be concerned with the determination of motions of this kind for compressible simple materials of the following general types: fluids, isotropic solids, and anisotropic solids. This problem was first posed in a paper by Ericksen [4] in which he derived a number of deformations which can be maintained in every incompressible homogeneous isotropic perfectly elastic solid by the action of surface tractions alone. In a later paper ERICKSEx [5] showed that any deformation which is controllable in compressible homogeneous isotropic perfectly elastic solids in equilibrium must necessarily be homogeneous. We use this result to show that any test motion which is to be controllable in compressible simple fluids, isotropic solids or anisotropic solids after some time $t$ must be homogeneous after that time. The details of the motion before time $t$ depend on the duration of memory of the particular type of material considered. These details are derived for compressible simple materials having perfect memory, finite memory, and fading memory. Generally speaking, depending on whether the memory is perfect, finite or fading, the deformation up to time $t$ must be homogeneous and respectively, uniformly accelerated, arbitrary or fixed. Once the motion has become controllable it must be homogeneous and have constant acceleration. This latter motion is discussed in the article by Truesdell and Noli $[3, \S 28]$. 
In Section 2 we present constitutive equations for compressible simple fluids and isotropic simple solids in forms which are most useful for our analysis. The field equations and a more explicit definition of the condition that a motion be controllable are given in Section 3. In Sections 4 and 5 we apply this condition to the determination of controllable motions in isotropic solids. The results obtained are summarized above. Section 6 contains a similar treatment for simple fluids. The final two sections are devoted to the extensions of results for isotropic solids to anisotropic solids. The proof of the extension is carried out in Section 7, using the result proved in Section 8, that the constitutive equation for a solid having any material symmetry can be represented in one of two general forms. In Section 8, we first review the method of constructing representations of constitutive equations. We then show that for any material symmetry, the corresponding representation can take one of the two general forms used in Section 7.

Notation. We refer the components of all tensors to a fixed CarTEsran coordinate system. We use the usual conventions of index notation. Repeated indices denote summation over the range $i=1,2,3$. A comma preceding an index denotes partial differentiation, i.e., $\varphi_{i}=\partial \varphi / \partial x_{i}$. A second rank tensor is denoted by the matrix $\mathbf{D}=\left\|D_{i j}\right\|$ of its components. $\mathbf{D}^{T}, \mathbf{D}^{-1}, \operatorname{tr} \mathbf{D}=D_{i i}$, det $\mathbf{D}=\left|D_{i j}\right|, \mathbf{D}^{N}$ denote, respectively, the transpose, inverse, trace, determinant and $N$ th power of $\mathbf{D}$. $\mathbf{D}_{\mathbf{0}}=\mathbf{1}=\left\|\delta_{i j}\right\|$ represents the identity matrix, where $\delta_{i j}$ is the KRonecker delta.

\section{The Constitutive Equation}

The motion of a deformable body can be described by specifying the motion of each of its particles. Relative to a fixed CARTESIAN coordinate system, the deformation of a body can be described by a relation

$$
\mathbf{x}=\mathbf{x}(\mathbf{X}, \tau)
$$

between the position $\mathbf{X}$ occupied by a generic particle in some reference configuration and the position $\mathbf{x}$ occupied at each time $\tau$ in some time interval. This relation is assumed to be one-to-one and at least three times continuously differentiable.

The deformation gradient at a particle taken with respect to some reference configuration is denoted by $\mathbf{F}(\mathbf{X}, \tau)=\left\|\partial x_{i} / \partial X_{j}(\mathbf{X}, \tau)\right\|$. For simple materials, the stress tensor $\boldsymbol{\sigma}(\mathbf{X}, t)$ depends on all the values of $\mathbf{F}(\mathbf{X}, t)$ in some time interval $[t-T, t]$, i.e., is a functional of the history $\mathbf{F}(\mathbf{X}, \tau)$,

$$
\boldsymbol{\sigma}(\mathbf{X}, t)=\underset{\tau=t-T}{\frac{t}{\mathfrak{T}}}[F(X, \tau)] .
$$

The interval $[t-T, t]$ defines the extent of this memory of the material.

A homogeneous simple solid is defined [1] as a simple material having a fixed reference configuration and such that the form of the response functional is independent of $\mathbf{X}$. 
If the simple solid is isotropic in its reference configuration, then this property and the Principle of Material Indifference [1] imply that (2.2) can be expressed in the form

$$
\boldsymbol{\sigma}(t)={\underset{\tau=t-T}{\mathfrak{T}}}_{\mathfrak{T}}^{t}\left[\mathbf{C}^{t}(\tau) ; \mathbf{B}^{-1}(t)\right],
$$

where $\mathfrak{T}$ is a functional of the history of the relative right CAUCHY-GREEN tensor $\mathbf{C}^{t}(\tau)$ defined by

$$
\mathbf{C}^{t}(\tau)=\left\|C^{t} t_{i j}(\tau)\right\|=\left\|\frac{\partial x_{p}(\tau)}{\partial x_{i}(t)} \frac{\partial x_{p}(\tau)}{\partial x_{j}(t)}\right\|,
$$

and a function of the inverse left $\mathrm{CACCHY}_{\text {-GReEN tensor }}$

$$
\mathbf{B}^{-1}(t)=\left\|B_{i j}{ }^{-1}(t)\right\|=\left\|\frac{\partial X_{q}}{\partial x_{i}(t)} \frac{\partial X_{q}}{\partial x_{j}(t)}\right\| .
$$

Although, in most applications the response functional is expressed in terms of the left CAUCHY-GREEN tensor $\mathbf{B}$, for our present purposes it is convenient to use its inverse $\mathbf{B}^{-1} . \sigma, \mathbf{C}^{t}(\tau), \mathbf{B}^{-1}$ depend on a fixed particle through its position $\mathbf{X}$ in the reference configuration. By means of (2.1) we can express them in terms of the position $\mathbf{x}(t)$ in the current configuration.

Dependence of $\mathfrak{I}$ on $\mathbf{B}^{-1}(t)$ represents the effect on the stress of the present deformation with respect to the fixed reference configuration. Dependence on the history $\mathbf{C}^{t}(\tau)$ represents the effect of the deformations which the material has already undergone.

An additional restriction on $\mathfrak{T}$ is the isotropy condition

$$
\begin{aligned}
& \mathbf{Q} \underset{\tau=t-T}{\mathfrak{Z}}\left[\mathbf{C}^{t}(\tau) ; \mathbf{B}^{-1}(t)\right] \mathbf{Q}^{T}=\underset{\tau=t-T}{\mathfrak{T}}\left[\mathbf{Q} \mathbf{C}^{t}(\tau) \mathbf{Q}^{T} ; \mathbf{Q} \mathbf{B}^{-1}(t) \mathbf{Q}^{T}\right], \\
& \mathbf{Q} \mathbf{Q}^{T}=\mathbf{Q}^{T} \mathbf{Q}=\mathbf{1}
\end{aligned}
$$

which must be satisfied identically in $\mathbf{C}^{t}(\tau)$ and $\mathbf{B}^{-1}(t)$ for all constant orthogonal transformations Q. A theory of general representations for response functionals $\mathfrak{I}$ satisfying $(2.6)$ has been developed by RIvLIN and his co-workers for various continuity assumptions. (See [6] for a resume of this work.) A specific example of a constitutive equation arising from such representations is given by

$$
\boldsymbol{\sigma}(t)=\varphi_{0} 1+\varphi_{1} \mathbf{B}^{-1}+\varphi_{2} \mathbf{B}^{-2}+\int_{t-T}^{t} \mathbf{C}^{t}(\tau) \theta(t-\tau) d \tau .
$$

The $\varphi_{N}$ are polynomials in the invariants of $\mathbf{B}^{-1}$, defined by

$$
I_{1}=\operatorname{tr} \mathbf{B}^{-1}, I_{2}=1 / 2\left[\left(\operatorname{tr} \mathbf{B}^{-1}\right)^{2}-\operatorname{tr} \mathbf{B}^{-2}\right], I_{3}=\operatorname{det} \mathbf{B}^{-1}
$$

and the invariant

$$
\omega(t)=\int_{t-T}^{t} \operatorname{tr} \mathbf{C}^{t}(\tau) \psi(t-\tau) d \tau
$$


$\mathbf{C}^{t}(\tau)$ can be taken as depending on $\mathbf{x}(t)$, implying that $\omega=\omega(\mathbf{x}(t), t)$. The scalar quantities $\varphi_{N}, \theta, \psi$ are material parameters, depending on the particular solid considered. $\theta$ and $\psi$ are assumed to be continuous on the interval $[t-T, t]$ whatever the choice of $T$. The response functional (2.7) may be made more general by adding integrals of greater multiplicity whose integrands are matrix products formed from $\mathbf{B}^{-1}, \mathbf{C}^{t}\left(\tau_{1}\right), \ldots, \mathbf{C}^{t}\left(\tau_{5}\right)$. However, (2.7) is sufficiently general for our purposes. For brevity, we will refer to materials of this type as isotropic solids.

A homogeneous simple fluid is a simple material whose reference configuration may be taken as the one at the current time $t$. Dependence of the response functional $(2.3)$ on $\mathbf{B}^{-1}(t)$ is replaced by dependence on the present density $\varrho(t)$. In addition, simple fluids are isotropic, so that their response functionals satisfy a condition of form (2.6). A representation of a constitutive equation for a compressible simple fluid which will be useful is

$$
\sigma=-p(\varrho) \mathbf{1}+\mathbf{1} \int_{t-T} \operatorname{tr} \mathbf{C}^{t}(\tau) \psi(t-\tau ; \varrho) d \tau+\int_{t-T^{\prime}}^{t} \mathbf{C}^{t}(\tau) \theta(t-\tau ; \varrho) d \tau .
$$

The scalar $p(\varrho)$ is the pressure the fluid would be supporting if it had remained at rest in its present configuration at all times in the past.

\section{Field Equations}

The body force field $\mathbf{f}$ per unit mass is assumed to be conservative. To be dynamically possible, the motion (2.1) of the material and the stress field related to it by (2.3) must satisfy the equations of motion

$$
\frac{\partial \sigma_{i j}(t)}{\partial x_{j}(t)}+\varrho(t) f_{i}=\varrho(t) \ddot{x}_{i}(t)
$$

in the region occupied by the body at each time $t$. $\varrho(t)$ is the density of the material at time $t$ which for simple solids is related to the density $\varrho_{0}$ in the reference configurations by

$$
\varrho(t)=I_{3}{ }^{1 / 2} \varrho_{0} .
$$

We are particularly interested in those motions which are possible in every compressible simple material of the types mentioned in Section 1 . By a controllable motion for materials of a given type, we mean a mapping (2.1) which is such that the system consisting of (3.1) and the appropriate constitutive equation is satisfied identically, independent of the properties of particular materials of that type. By controllable tensors we mean the deformation tensors $\mathbf{B}^{-1}$ and $\mathbf{C}^{t}(\tau)$ corresponding to a controllable motion.

When the system consisting of (3.1) and the constitutive equation (2.7) is restricted by the condition that the motion be controllable, we obtain a system of equations for the controllable tensor fields $\mathbf{B}^{-1}(\mathbf{x}(t), t)$ and $\mathrm{C}^{t}(\mathbf{x}(t), \tau)$ for some range of $\tau$. It is seen from (2.4), (2.5) that both 
of these strain tensors can be interpreted as the covariant components of metric tensors. Thus, in order that $\mathbf{B}^{-1}(t)$ lead to a one-to-one mapping (2.1) at time $t$, the Riemann-Christoffere tensor based on $\mathbf{B}^{-1}$ must vanish. Similarly, in order that $\mathbf{C}^{t}(\tau)$ lead to a one-to-one relation between $\mathbf{x}(\tau)$ and $\mathbf{x}(t)$, the RiemanN-Christoffiel tensor based on $\mathbf{C}^{t}(\tau)$ must vanish for each desired choice of $\tau$. An explicit statement of this condition, not needed for our purposes, is given in [5].

\section{Determination of the General Controllable Motion}

We now consider motions of isotropic solids which are to be controllable at time $t$. Such a motion must be possible in the particular isotropic solid whose constitutive equation is given by (2.7). Substitution of this constitutive equation and (3.2) into (3.1) yields the following equation

$$
\begin{aligned}
\sum_{N=0}^{2} \varphi_{N} B_{i j, j}^{-N} & +\sum_{N=0}^{2} \sum_{M=1}^{3} \frac{\partial \varphi_{N}}{\partial I_{M}} I_{M, j} B_{i j}^{-N}+ \\
& +\sum_{N=0}^{2} \frac{\partial \varphi_{N}}{\partial \omega} B_{i j}^{-N} \int_{t-T}^{t} C^{t} p p, j(\tau) \psi(t-\tau) d \tau+ \\
& +\int_{t-T}^{t} \theta(t-\tau) C^{t} i j, j(\tau) d \tau+\varrho_{0} I_{3}^{1 / 2} f_{i}=\varrho_{0} I_{3}^{1 / 2} \ddot{x}_{i} .
\end{aligned}
$$

(4.1) must hold for arbitrary choices of the material parameters $\varphi_{N}$, $\partial \varphi_{N} / \partial I_{M}, \partial \varphi_{N} / \partial \omega, \psi, \theta$, and $\varrho_{0}$. From the arbitrariness of $\varrho_{0}$ and the condition that $I_{3}=\operatorname{det} \mathbf{B}^{-1} \neq 0$ in a continuous motion, we conclude that a necessary condition that a motion be controllable at time $t$ is that

$$
\ddot{x}_{i}(t)=f_{i} \text {. }
$$

Continued application of this argument implies that the coefficients of $\varphi_{N}, \partial \varphi_{N} / \partial I_{M}$, and $\partial \varphi / \partial \omega$ must vanish, yielding the conditions

$$
\begin{gathered}
B_{i j, j}^{-N}=0, \quad I_{M, j} B_{i j}^{-N}=0, \quad\left(\begin{array}{l}
N=0,1,2 \\
M=1,2,3
\end{array}\right), \\
B_{i j} \int_{t-T}^{t} \psi(t-\tau) C_{p p, j}^{t}(\tau) d \tau=0,(N=0,1,2), \\
\int_{t-T}^{t} \theta(t-\tau) C^{t}{ }_{i j, j}(\tau) d \tau=0 .
\end{gathered}
$$

Letting $N=1$ in $(4.3)_{1}$ and $N=0, M=1$ in $(4.3)_{2}$ and using (2.8) we obtain as conditions on $\mathbf{B}^{-1}$ that

$$
B_{i j, j}^{-1}(t)=0, B_{i i, j}^{-1}(t)=0 .
$$


Letting $N=0$ in (4.4) we find

$$
\int_{t-T}^{t} \psi(t-\tau) C^{t} t_{i i, j}(\tau) d t=0 .
$$

For a controllable motion, the strain history $\mathbf{C}^{t}(\tau)$ must be the same for all isotropic solids. (4.5) and (4.7), with $C^{t}{ }_{i j, j}(\tau)$ and $C^{t}{ }_{i i, j}(\tau)$ fixed, must be satisfied for each choice of the material functions $\theta$ and $\psi$. By an argument similar to that used to prove the Fundamental Lemma of the Calculus of Variations [7, p. 185], this implies that any motion which is to be controllable at time $t$ must satisfy

$$
C^{t} i j, j(\tau)=0, C^{t} i i, j(\tau)=0
$$

for $\tau$ in $[t-T, t]$.

In determining the deformations which can be maintained in every compressible isotropic homogeneous prefectly elastic solid in equilibrium, ERICKSEN [5] also showed that the finite strain tensor $\mathbf{B}^{-1}$ must satisfy the system $(4: 6)$. He then showed that a strain tensor whose components are of the form of the covariant components of a metric tensor, which satisfies a system such as (4.6) or (4.8) and whose Riemann-Christoffer tensor vanishes, is a constant tensor field. Thus, recalling the discussion at the end of Section 3, we conclude that $\mathbf{B}^{-1}(t)$ is a constant tensor field and so is $\mathbf{C}^{t}(\tau)$ for each $\tau$ in $[t-T, t]$. The system (4.3), (4.4), (4.5) is now satisfied as would any system which would arise from a constitutive equation more complex than (2.7).

A necessary and sufficient condition that $\mathbf{B}^{-1}(t)$ be a constant tensor field is that time $t(2.1)$ have the form

$$
\mathbf{x}(\mathbf{X}, t)=\widehat{\mathbf{F}}(t) \mathbf{X}+\widehat{\mathbf{b}}(t), \operatorname{det} \widehat{\mathbf{F}}(t) \neq 0,
$$

while a necessary and sufficient condition that $\mathbf{C}^{t}(\tau)$ be a constant tensor field for $\tau$ in $[t-T, t]$ is that

$$
\mathbf{x}(\mathbf{x}(t), \tau)=\overline{\mathbf{F}}(\tau) \mathbf{x}(t)+\overline{\mathbf{b}}(\tau), \operatorname{det} \overline{\mathbf{F}}(\tau) \neq 0,
$$

for $\tau$ in $[t-T, t]$. The result of combining (4.9) and (4.10) is stated in the following theorem.

Theorem 1. A necessary condition that a motion be controllable at time $t$ in isotropic solids is that the motion (2.1) have the form

$$
\mathbf{x}(\mathbf{X}, \tau)=\mathbf{F}(\tau) \mathbf{X}+\mathbf{b}(\tau), \operatorname{det} \mathbf{F}(\tau) \neq 0,
$$

for $\tau$ in $[t-T, t]$.

\section{Controllable Motions in Isotropic Solids}

We now investigate the restrictions on (4.11) due to (4.2) and various durations of memory. For the present, we assume that the controllable motions are to be produced in solids which are initially at rest in their 
reference configurations. Then, (2.1) has the form

$$
\mathbf{X}(\mathbf{X}, \tau)=\mathbf{X}, \tau \leqslant 0 .
$$

A motion which is to be controllable in every isotropic solid must be controllable in those having perfect memory. For such solids, the deformations in the entire interval $[0, t]$ determine the present stress. Hence the results of Section 4 must hold for $T=t$. In particular, by Theorem 1 , (2.1) must have the form (4.11) for $\tau$ in $[0, t]$.

Let $t^{*}$ be an arbitrary time such that $t^{*}<t$. It follows from $(2.4)$, (2.5), and (4.11) that $\mathbf{B}^{-1}\left(t^{*}\right)$ and the history $\mathbf{C}^{t *}(\tau)$ are constant tensor fields for $\tau$ in $\left[0, t^{*}\right]$. (2.7) then implies that $\sigma_{i j, j}\left(t^{*}\right)=0$. Since (3.1) must hold for each choice of $t^{*}$ in $[0, t]$, we conclude that (4.2) must hold on the entire interval $[0, t]$.

From (4.11) and the condition that $\mathbf{F}(\tau)$ must be nonsingular in $[0, t]$, we obtain the acceleration field at time $\tau$.

$$
\ddot{x}(\mathbf{x}(\tau), \tau)=\ddot{\mathbf{F}}(\tau) \mathbf{F}^{-1}(\tau)[\mathbf{x}(\tau)-\mathbf{b}(\tau)]+\ddot{\mathrm{b}}(\tau) .
$$

Comparing (4.2) at time $\tau$ with (5.2), we see that a conservative body force field is compatible with this acceleration field if and only if $\mathbf{f}$ has the form

$$
\mathbf{i}=\mathbf{G} \mathbf{x}+\mathbf{g}
$$

where $\mathbf{g}$ is a vector field and $\mathbf{G}$ is a symmetric tensor field, both independent of $x$. Since the most physically meaningful form of (5.3) is a constant body force field, we take $\mathbf{G}=\mathbf{0}$ and $\mathbf{g}=$ constant here and throughout the paper. Substituting (5.2) and (5.3) with $G=0$ in (4.2), we conclude that

$$
\ddot{\mathbf{F}}=\mathbf{0}, \ddot{\mathrm{b}}=\mathbf{g}
$$

for $\tau$ in $[0, t]$. (4.11), (5.1), and (5.4) now imply that a controllable motion must have the general form

$$
\mathbf{x}(\mathbf{X}, \tau)=\left(\mathbf{F}_{1} \tau+\mathbf{1}\right) \mathbf{X}+\mathbf{g} \frac{\tau^{2}}{\mathbf{2}}+\mathbf{a} \tau, \tau \text { in }[0, t]
$$

where $\mathbf{a}$ and $\mathbf{F}_{1}$ are constant vector and tensor fields, respectively. $\mathbf{F}_{1}$ represents an initial uniform velocity gradient field. It is clear from (2.3), (2.4), (2.5), (3.1), and (5.3) with $G=0$, that a motion of form (5.5) is possible in any simple solid.

For some materials, it may be possible to establish the homogeneous motion (5.5) with an initial uniform velocity gradient by appropriate surface tractions. An example would be a pure shearing deformation suddenly imposed on a material with no instantaneous elasticity, a Katurn-type material. Because the materials under consideration are compressible, it does not seem generally possible to produce instantaneously an initial uniform velocity gradient field by the action of surface tractions alone. Since we are considering motions possible in all isotropic solids, we require that any controllable motion begin continuously from the 
initial state (5.1). Combining this requirement with the above results, we conclude the following theorem.

Theorem 2. Let $g$ be a constant body force field and let (5.1) hold for $t \leqslant 0$. Let the class of materials considered be isotropic solids having perfect memory on $[0, t]$. If a motion is to be controllable at time $t$ in materials of this class, and be continuously initiated, then it must be uniformly accelerated motion, in which case (2.1) reduces to

$$
\mathbf{x}(\mathbf{X}, \tau)=\mathbf{X}+\mathrm{g} \frac{1}{2} \tau^{2}, \quad \tau \text { in }[0, t] .
$$

By considering controllable motions in the narrower class of isotropic solids having a finite memory of duration $T$, we can avoid the above restrictions arising from the condition that the motion be continuously initiated. For such solids, the stress at time $t$ is determined by the deformation in the interval $[t-T, t]$, for some finite $T>0$.

Suppose a motion is initiated at time $\tau=0$ in an isotropic solid at rest for $t \leqslant 0$. If we require that this motion be controllable at a time $t^{*}<T$, then the material has a memory for the entire interval $\left[0, t^{*}\right]$. By 'Theorem 2 the only continuous controllable motion is given by (5.6). Consequently, for isotropic solids with finite memory of duration $T$, we require that a motion initiated at $\tau=0$ be controllable only at time $t>T$ when the details of the initiation of the motion cannot influence the present stress.

Let $t$ now be the earliest time at which the motion is controllable, i.e., at which (4.1) is satisfied identically independently of the particular solid considered. (4.2) must hold for all times after $t$. By Theorem $1,(2.1)$ must have the form (4.11) for each $\tau$ in the interval $[t-T, t]$. Now, let $t^{*}$ be a fixed time in the open interval $(t-T, t)$. (2.4), (2.5), and (4.11) imply that $\mathbf{B}^{-1}\left(t^{*}\right)$ and $\mathbf{C}^{* *}(\tau)$ are constant tensor fields for $\tau$ in $\left[t-T, t^{*}\right]$. If the motion (2.1) is homogeneous during the extended interval $\left[t^{*}-T, t^{*}\right]$ then $\mathbf{C}^{* *}(\tau)$ is a constant tensor field for $\tau$ in $\left[t^{*}-T, t^{*}\right]$. By (2.3), (2.4), (2.5), $\sigma$ must be a constant tensor field for each $\tau$ in $\left[t^{*}, t\right]$, during which the motion will have to satisfy (4.2). Therefore, the motion is controllable at time $t^{*}$. Since we assumed that $t$ is the earliest time at which the motion is controllable, we conclude that (2.1) must be nonhomogeneous for $\tau<t-T$. Consequently for $t^{*} \leqslant t, \sigma\left(t^{*}\right)$ is not a constant field, and (4.2) does not hold.

For $\tau \geqslant t,(4.2)$ and (4.11) hold. Assuming a constant body force field, our previous argument shows that $\mathbf{F}$ and $\mathbf{b}$ must satisfy (5.4) on this interval. Integrating these conditions, we find that a motion which is controllable for $\tau \geqslant t$ must have the form

$$
\mathbf{x}(\mathbf{X}, \tau)=\left(\mathbf{F}_{\mathbf{0}}+\mathbf{F}_{1} \tau\right) \mathbf{X}+\mathbf{g} \frac{\tau^{2}}{2}+\mathbf{b} \tau+\mathbf{e},
$$

where $\mathbf{F}_{0}, \mathbf{F}_{1}$, and $\mathbf{b}$, $\mathbf{c}$ are constant tensor and vector fields, respectively.

The above results are summarized in the following theorem. 
Theorem 3. Let $g$ be a constant body force field and (5.1) hold for $\tau \geqslant 0$. Let the class of materials consist of isotropie solids having finite memory of duration $T$. In order that a motion be controllable at time $t$ and be nonrigid in materials of this class, it is necessary and sufficient that $t>T$ and that (2.1) be defined as follows,

$$
\begin{aligned}
& \mathbf{x}(\mathbf{X}, \tau) \ldots \text { continuous and arbitrary, } \quad 0 \leqslant \tau \leqslant t-T, \\
& =\mathbf{F}(\tau) \mathbf{X}+\mathbf{b}(\tau), \operatorname{det} \mathbf{F}(\tau) \neq 0, \quad t-T \leqslant \tau \leqslant t, \\
& =\left(\mathbf{F}_{0}+\mathbf{F}_{\mathbf{1}} \tau\right) \mathbf{X}+\mathbf{g}(\tau), \operatorname{det}\left(\mathbf{F}_{\mathbf{0}}+\mathbf{F}_{1} \tau\right) \neq 0, \quad \tau \geqslant t
\end{aligned}
$$

where $\mathbf{F}_{\mathbf{0}}, \mathbf{F}_{1}$ are constant tensors, $\mathbf{g}(\tau)$ is the rigid body motion part of $(5.7)$, and $\mathbf{F}(\tau), \mathbf{b}(\tau)$ are continuous tensor and vector valued functions of $\tau$ satisfying (5.4) at $\tau=t$. The only restriction on the motion in the interval $[0, t-T]$ is that it lead to an homogeneous motion during the interval $[t-T, t]$.

The necessity of the form (5.8) for the motion follows from the discussion preceding (5.7). Its sufficiency is readily established from (2.3), (2.4), (2.5), (3.1), (5.4), and (5.8). An analysis of the condition $\operatorname{det}\left(\mathbf{F}_{\mathbf{0}}+\mathbf{F}_{1} \tau\right) \neq 0$ has been given by Truesdell and Nolu [3, $\left.\$ 28\right]$.

Finally, we consider isotropic solids having fading memory. For these materials the deformations in the recent past influence the present stress $\sigma(t)$ more strongly than those in the distant past. In particular, in a material with fading memory, the manner in which a motion is initiated has diminishing influence on $\sigma(t)$ as $t$ increases. Thus, the material has a perfect memory for all deformations in any finite interval after the motion is initiated, during which the only continuous controllable motion is the rigid one (5.6), by Theorem 2. However, the previous results suggest that if a homogeneous motion were to be established and maintained, then it would become controllable a long time after its initiation, when the influence of the details of its initiation on $\sigma(t)$ becomes negligible.

One formulation of the property of fading memory has been given by Coleman and Nold [8]. This formulation leads to an integral approximation to the response functional in (2.3) which contains (2.7) as a special case when $T \rightarrow \infty$ and $\varphi_{N}$ depends linearly on the invariant $\omega(t)$ defined in (2.9). Futrhermore the material functions $\theta(s), \psi(s)$ have the property that they tend to zero as $s \rightarrow \infty$. We assume that $\theta$ and $\psi$ tend to zero sufficiently rapidly so that the integrals in (4.1) converge uniformly. We can then justify interchanging differentiation and integration.

The analysis and results of Section 4 now apply. In particular, (4.11) holds for $\tau$ in $(-\infty, t)$. Let $t_{1}$ be an arbitrary time in this interval. It follows from (2.4), (2.5), and (4.11) that $\mathbf{B}^{-1}\left(t_{1}\right)$ and $\mathbf{C}^{t 1}(\tau)$ are constant strain fields for $\tau$ in $\left(-\infty, t_{1}\right)$. Furthermore, the general constitutive equation (2.3), with $T$ taken as $\infty$, shows that the stress field $\sigma\left(l_{1}\right)$ is constant. This, together with (3.1) implies that (4.2) holds at $\tau=t_{1}$. Since $t_{1}$ was chosen arbitrarily, (4.2) must hold on the entire interval $(-\infty, t]$. Applying (4.2) to (4.11), we conclude that the motion has the form (5.7) on $(-\infty, t]$. From (2.4) and (4.7) we see that $\mathbf{C}^{t}(\tau)$ becomes 
unbounded as $\tau \rightarrow-\infty$, i.e., the distance between particles in previous configurations becomes arbitrarily large compared to this distance in the current configuration. Since for some solids the deformation gradients must be bounded, we conclude the following result.

Theorem 4. Let $\mathrm{g}$ be a constant body force field. A necessary and sufficient condition that a motion be controllable in isotropic solids having fading memory is that (2.1) have the form

$$
\mathbf{x}(\mathbf{X}, \tau)=\mathbf{F}_{0} \mathbf{X}+\mathbf{g}(\tau), \tau \text { in }(-\infty, t]
$$

where $\mathbf{F}_{0}$ is a constant tensor, and $\mathbf{g}(\tau)$ is defined as in (5.8).

In other terms, ignoring the effects of gravity the only possible controllable motion in an isotropic solid with fading memory is one in which the material is held in a fixed deformed state. In other terms the only test situation common to all isotropic solids with fading memory is a stress relaxation test.

\section{Simple Fluids}

The analysis for simple fluids is similar to that for isotropic solids. A motion which is to be controllable at time $t$ in homogeneous compressible simple fluids must be possible in the particular fluid whose constitutive equation is given by (2.10). The condition that a motion be controllable is obtained by substituting (2.10) into (3.1),

$$
\begin{aligned}
& \int_{t-T}^{t} \mathbf{C}_{p p, i}(\tau) \psi(t-\tau ; \varrho) d \tau+\varrho, i \int_{t-T}^{t} \mathbf{C}_{p p}^{t}(\tau) \frac{\partial \psi}{\partial \varrho}(t-\tau ; \varrho) d \tau+ \\
& +\int_{t-T}^{t} \mathbf{C}_{i j, j}(\tau) \theta(t-\tau ; \varrho) d \tau+\varrho, j \int_{t-T}^{t} \mathbf{C}_{i j}(\tau) \frac{\partial \theta}{\partial \varrho}(t-\tau ; \varrho) d \tau- \\
& -\frac{d p}{d \varrho} \varrho_{i}+\varrho f_{i}=\varrho \ddot{x}_{i} .
\end{aligned}
$$

In order that a motion be controllable at time $t,(6.1)$ must hold for arbitrary choices of the material parameters $\varrho, p(\varrho), \psi, \theta, \partial \psi / \partial \varrho, \partial \theta / \partial \varrho$. Arguing as in Section 4, we conclude that (4.2) must hold at times beginning with $t$, while the following system holds for $\tau$ in the interval $[t-T, t]$

$$
\begin{aligned}
& C^{t}{ }_{i i, j}(\tau)=0, \quad C^{t}{ }_{i j, j}(\tau)=0, \\
& C^{t}{ }_{i i}(\tau) \varrho, j=0, \quad C^{t}{ }_{i j}(\tau) \varrho, j=0, \quad \varrho, i=0 .
\end{aligned}
$$

The system (6.2) is the same as the system (4.8). Requiring the RimmañChristoffel tensor based on $\mathbf{C}^{t}(\tau)$ to vanish for each $\tau$ in $[t-T, t]$, "we find that $\mathbf{C}^{t}(\tau)$ must be a constant strain tensor field so that on this interval the motion has the form $(4.10)$.

Considering now the system (6.3), we see that it is satisfied if and only if $\varrho_{i}(l)=0$. Applying this condition to the equation of conservation 
of mass

$$
\varrho(t)=\varrho(\tau)\left|\frac{\partial x_{i}(\tau)}{\partial x_{j}(t)}\right|,
$$

and observing that the JACOBIAN of the mapping (4.10) must be independent of the coordinates $x_{i}(t)$ and nonzero, we conclude that $\partial \varrho(\tau) / \partial x_{i}(\tau)=0$ for $\tau$ in $[t-T, t]$. By (4.10) and (6.4)

$$
\varrho(\tau)=\varrho(t)(\operatorname{det} \mathbf{F}(\tau))^{-1} \text {. }
$$

We now turn to the restrictions on these motions implied by the range of memory involved. For the present, we assume the fluids are initially at rest,

$$
\mathbf{x}(\mathbf{x}(t), \tau)=\overrightarrow{\mathbf{x}}(\mathbf{x}(t)), \quad \tau \leqslant 0 .
$$

Some fluids may have a perfect memory over any finite time interval, that is, the duration of memory $T$ equals the present time $t$. Therefore, (4.10) must hold on $[0, t]$. Let $t_{1}$ be some instant in this interval. By arguments similar to those used for the discussion of isotropic solids having perfect memory, we conclude that $\mathbf{C}^{t 1}(\tau)$ is a constant strain tensor field for $\tau$ in $\left[0, t_{1}\right]$. It follows that $\sigma\left(t_{1}\right)$ is also a constant field. Since $t_{1}$ is arbitrary, (4.2) must hold on $[0, t]$. Again allowing at most a constant body force field $g$, we are led to the conclusion that in (4.10)

$$
\ddot{\mathbf{F}}(\tau)=\mathbf{0}, \ddot{\mathbf{b}}(\tau)=\mathbf{g} .
$$

These restrictions, along with

$$
\mathbf{x}(\mathbf{x}(t), t)=\mathbf{x}(t),
$$

imply that (4.10) must have the form

$$
\mathbf{x}(\mathbf{x}(t), \tau)=\left[\mathbf{1}-(t-\tau) \mathbf{F}_{1}\right] \mathbf{x}(t)+\mathbf{g} \frac{\mathbf{1}}{2}\left(\tau^{2}-t^{2}\right)+\mathbf{c}(\tau-t),
$$

where $\mathbf{F}_{1}$ is a constant tensor. $\mathbf{F}_{1}$ represents a uniform velocity gradient at time $t$. From (6.9) we find that the initial velocity gradient field is $\mathbf{F}_{1}\left[\mathbf{1}-t \mathbf{F}_{1}\right]^{-1}$. Since in some compressible fluids the motion must begin continuously, we require that this field vanish. As (6.9) is not defined at $\tau=0$ if $\left[\mathbf{1}-t \mathbf{F}_{\mathbf{1}}\right]^{-1}=0$, we conclude that $\mathbf{F}_{\mathbf{1}}=\mathbf{0}$.

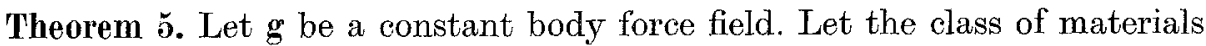
considered be simple fluids having perfect memory on $[0, t]$. In addition, let (6.6) hold for $\tau \leqslant 0$. Then a continuous motion is controllable at time $t$ in materials of this class if and only if it is uniformly accelerated motion, that is, (2.1) has the form

$$
\mathbf{x}(\mathbf{x}(t), \tau)=\mathbf{x}(t)+\mathbf{g}\left(\frac{\tau^{2}-t^{2}}{2}\right) .
$$

We now consider fluids having finite memory of duration $T$. The determination of the controllable rnotions for this case is similar to that for the corresponding isotropic solid except that the appropriate reference 
configuration is always the current configuration. Let $t$ be the present time and $t_{2}$ be an earlier time at which the motion first becomes controllable. There will be no loss in generality if $t_{2}$ is in $[t-T, t]$. By the same argument as was used for isotropic solids with finite memory, we conclude that the controllable motions will be nonrigid and continuous if and only if $t_{2}>T$. Furthermore, for $\tau$ in $\left[0, t_{1}-T\right]$ the motion is arbitrary, while for $\tau$ in $\left[t_{2}-T, t_{2}\right],(6.2)$ implies the form

$$
\mathbf{x}\left(\mathbf{x}\left(t_{2}\right), \tau\right)=\overline{\mathbf{F}}(\tau) \mathbf{x}\left(t_{2}\right)+\overline{\mathbf{b}}(\tau) .
$$

Since the motion is to be controllable at all times greater than $t_{2}$, we conclude that it must have form (4.10) on the extended interval $\left[t_{2}-T, t\right]$, while (4.2) holds on $\left[t_{2}, t\right]$. Assuming that the body force field is constant, this last condition implies that $\mathbf{x}[\mathbf{x}(t), \tau]$ has the form $(6.9)$ on $\left[t_{2}, t\right]$.

The following theorem combines these results.

Theorem 6. Let $\mathrm{g}$ be a constant body force field and (6.6) hold for $\tau ₹ 0$. Let the class of materials considered consist of simple fluids having finite memory of duration $T$. A motion will become controllable at time $t_{2}<t$ and be nonrigid in materials of this class if and only if (i) $t_{2}>T$ and (ii) the motion has the structure

$$
\begin{aligned}
& \mathbf{x}[\mathbf{x}(t), \tau] \ldots \text { arbitrary and continuous, } \quad 0 \leqslant \tau \leqslant t_{2}-T, \\
& \quad=\mathbf{F}(\tau) \mathbf{x}(t)+\mathbf{b}(\tau), \operatorname{det} \mathbf{F}(\tau) \neq 0, \quad t_{2}-T \leqslant \tau \leqslant t_{2}, \quad \text { (6.12) } \\
& \quad=\left(\mathbf{1}-(t-\tau) \mathbf{F}_{1}\right) \mathbf{x}(t)+\mathbf{g}(\tau) \text {, det }\left[\mathbf{1}-(t-\tau) \mathbf{F}_{1}\right] \neq 0, t_{2} \leqslant \tau \leqslant t,
\end{aligned}
$$

where $\mathbf{F}_{1}$ is a constant tensor and $g(\tau)$ represents uniformly accelerated rigid motion. The corresponding density variation on $\left[t_{2}, t\right]$ is

$$
\varrho(\tau)=\varrho(t) \operatorname{det}\left[\mathbf{1}-(t-\tau) \mathbf{F}_{1}\right]^{-1} .
$$

This last result follows from (6.5) and (6.12).

The discussion for fluids with fading memory is also analogous to that for solids. An analysis leads to the conclusion that both (4.2) and (4.10) hold on the interval $(-\infty, t]$. The controllable motion then has form (6.9) on this interval. In order that this motion be defined it is necessary that for $\tau$ in $(-\infty, t]$.

$$
\begin{aligned}
\operatorname{det}\left[\mathbf{1}-(t-\tau) \mathbf{F}_{1}\right]= & 1+(\tau-t) \operatorname{tr} \mathbf{F}_{1}+\frac{(\tau-t)^{2}}{2}\left[\left(\operatorname{tr} \mathbf{F}_{1}\right)^{2}-\operatorname{tr} \mathbf{F}_{1}^{2}\right]+ \\
& +(\tau-t)^{3} \operatorname{det} \mathbf{F}_{1} \neq 0 .
\end{aligned}
$$

This is possible only if the set of proper numbers $1 /(\tau-t)$ of $\mathbf{F}_{1}$ consists of 0 and two complex numbers, or 0,0 and $0^{2}$. The first possibility requires that $\operatorname{det} \mathbf{F}_{1}=0$ and $\left(\operatorname{tr} \mathbf{F}_{1}\right)^{2}<2\left[\left(\operatorname{tr} \mathbf{F}_{1}\right)^{2}-\operatorname{tr} \mathbf{F}_{1}^{2}\right]$. However, it follows from (6.13) and (6.14) that as $\tau \rightarrow-\infty, \varrho \rightarrow 0$, which is not physically meaningful. The second possibility requires that $\operatorname{tr} \mathbf{F}_{1}=\operatorname{tr} \mathbf{F}_{1}{ }^{2}=\operatorname{det} \mathbf{F}_{1}=$ $=0$, in which case $\operatorname{det}\left[\mathbf{1}-(t-\tau) \mathbf{F}_{1}\right]=1$ and $\varrho(\tau)=\varrho(t)$. An example of such a motion is steady simple shearing flow.

${ }^{2}$ See $[3, \S 28]$. 
Theorem 7. Let $g$ be a constant body force field and the simple fluid have fading memory. A necessary and sufficient condition that a motion be controllable in fluids of this class is that it have the form (6.9) on the interval $(-\infty, t]$ and be isochoric.

\section{Anisotropic Simple Solids}

The results obtained in Section 5 for isotropic simple solids can be readily extended to include transversely isotropic solids and solids belonging to one of the crystal classes.

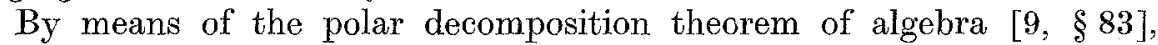
the nonsingular deformation gradient $\mathbf{F}(t)$ defined in Section 2 can be decomposed into the product of an orthogonal transformation $\mathbf{R}(t)$ and positive definite symmetric matrices $\mathbf{U}(t), \mathbf{V}(t)$ as follows,

$$
\begin{aligned}
& \mathbf{F}(t)=\mathbf{R}(t) \mathbf{U}(t)=\mathbf{V}(t) \mathbf{R}(t) \\
& \mathbf{R}(t) \mathbf{R}^{T}(t)=\mathbf{1}, \\
& \mathbf{U}(t)=\mathbf{U}(t)^{T}, \mathbf{V}(t)=\mathbf{V}(t)^{T} .
\end{aligned}
$$

$\mathbf{R}$ represents the rigid rotation of the neighborhood of a particle at time $t$ relative to its position in the reference configuration. $\mathbf{U}(t), \mathbf{V}(t)$ represent pure deformations.

The constitutive equation (2.2), when subjected to the restrictions of the Principle of Material Indifference, reduces to the form

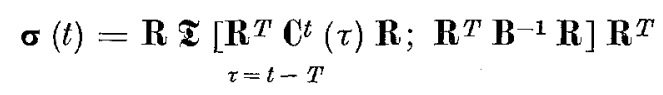

where $\mathbf{R}=\mathbf{R}(t)$. We will use the notation

$$
\overline{\mathbf{C}}^{t}(\tau)=\mathbf{R}^{T} \mathbf{C}^{t}(\tau) \mathbf{R}, \overline{\mathbf{B}}=\mathbf{R}^{T} \mathbf{B}^{-1} \mathbf{R} .
$$

The symmetry properties for any simple solid are defined by a group of orthogonal transformations $\{H\}$, called a material symmetry group. For example, for transversely isotropic materials, the group consists of rotations in a plane and possibly a reflection about an axis perpendicular to or lying in the plane. The material symmetry property for a given material implies that the response functional $\mathfrak{I}$ satisfies the condition

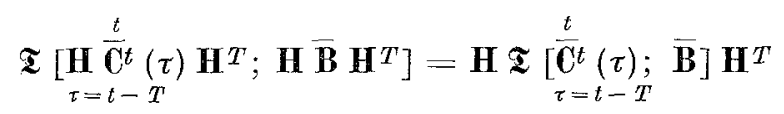

identically in $\overline{\mathbf{C}}^{t}(\tau)$ and $\overline{\mathbf{B}}$ for each transformation $\mathbf{H}$ in the group $\{H\}$ of symmetry transformations. A response functional satisfying (7.4) is said to be form-invariant under the transformations of the symmetry group $\{H\}$.

The theory of representations for form invariant response functionals has been developed by RIVLIN and his co-workers. In the present section we will state and use the form of the constitutive equation for a given 
anisotropic material which is most convenient for our purposes. In the next section we shall indicate its derivation.

For transversely isotropic materials and materials of the cubic, tetragonal and hexagonal systems, there is no loss in generality if the response functional is written in the form

$$
\mathfrak{I}=\varphi^{(1)} \mathbf{1}+\varphi^{(2)} \overline{\mathbf{B}}+\varphi^{(3)} \overline{\mathbf{B}}^{2}+\int_{t-T}^{t} \overline{\mathbf{C}}^{t}(\tau) \theta(t-\tau) d \tau+\boldsymbol{C}
$$

where $\theta$ is a scalar function of $t-\tau$. The tensor $c$ depends on certain combinations of $\overline{\mathbf{B}}$ and $\overline{\mathbf{C}}^{t}(\tau)$ which are appropriate only to the specific symmetry group under consideration. $\varphi^{(\alpha)}$ depends on the invariants (2.8), (2.9) and other scalar invariants appropriate to the given group. Substituting (7.5) into (7.2) and using (7.1) and (7.3) we see that the constitutive equation for a typical material having one of the above-mentioned symmetries has the form

$$
\begin{gathered}
\boldsymbol{\sigma}(t)=\varphi^{(1)} \mathbf{1}+\varphi^{(2)} \mathbf{B}^{-1}+\varphi^{(3)} \mathbf{B}^{-2}+\int_{t-T}^{t} \mathbf{C}^{t}(\tau) \theta(t-\tau) d \tau+ \\
+\mathbf{R}(t) \mathbf{C} \mathbf{R}^{T}(t) .
\end{gathered}
$$

In order to determine the controllable motions for simple solids having the above mentioned symmetries, the system of equations (3.1) and (7.6) must be satisfied identically, independently of the nature of the material functions $\varphi^{(1)}, \varphi^{(2)}, \varphi^{(3)}, \theta$, and those contained in $\boldsymbol{C}$. As in Section 4 , the application of this restriction to the system (3.1), (7.6) leads to a new system of equations among which are (4.2) and (4.3)-(4.5). We can thus now apply precisely the same analysis as was used for isotropic solids. Since the only controllable motions will be homogeneous motions, the equations in the above system arising from the terms $\mathbf{R}(t) \in \mathbf{R}^{T}(t)$ will be identically satisfied. Thus, all the results of Section 5 apply to materials having transverse isotropy, or belonging to the cubic, tetragonal or hexagonal systems.

For materials of the triclinic, monoclinc, and rhombic systems, the possible response functionals are written most usefully in the form

$$
\mathfrak{I}=\varphi \mathbf{1}+\mathfrak{T}^{*}
$$

where $\mathfrak{I}^{*}$ represents terms depending on certain combinations of $\overline{\mathbf{B}}$ and $\overline{\mathbf{C}}^{t}(\tau)$ which are appropriate to the specific symmetry group under consideration. $\varphi$ is a scalar material function in which the components $\overline{\mathbf{B}}_{i j}$ or their squares $\left(\overline{\mathbf{B}}_{i j}\right)^{2}$ appear as arguments. $\varphi$ also depends on the components $\bar{C}^{t}{ }_{i j}(\tau)$ or their squares $\left[\bar{C}^{t}{ }_{i j}(\tau)\right]^{2}$ by means of a combination of the functionals 


$$
\begin{gathered}
\omega_{i j}{ }^{(1)}=\int_{t-T^{\prime}}^{t} \bar{C}_{i j}(\tau) \psi_{i j}^{(1)}(t-\tau) d \tau \\
\omega_{i j}{ }^{(2)}=\int_{t-T}^{t} \int_{t-T}^{t} \psi_{i j}{ }^{(2)}\left(t-\tau_{1}, t-\tau_{2}\right) \bar{C}_{i j}\left(\tau_{1}\right) \bar{C}^{t}{ }_{i j}\left(\tau_{2}\right) d \tau_{1} d \tau_{2}
\end{gathered}
$$

appropriate to the group considered.

Proceeding as before, we find that a motion will be controllable only if

$$
\frac{\partial \bar{B}_{i j}}{\partial x_{k}(t)}=0, \quad \frac{\partial \bar{C}_{i j}(\tau)}{\partial x_{k}(t)}=0 .
$$

We note from (7.1) and (7.3) that $\overline{\mathbf{B}}=\mathbf{C}^{-1}=\mathbf{F}^{-1}\left(\mathbf{F}^{-1}\right)^{T}$. (7.9) ${ }_{1}$ now implies that the right $\mathrm{C}_{A U C H Y}$-Green strain tensor $\mathbf{C}=\mathbf{F}^{T} \mathbf{F}$ is a constant tensor field with respect to the reference configuration, i. e.,

$$
\frac{\partial}{\partial \bar{X}_{k}} C_{i j}=\frac{\partial}{\partial X_{k}}\left(\frac{\partial x_{p}(t)}{\partial X_{i}} \frac{\partial x_{p}(t)}{\partial \bar{X}_{j}}\right)=0 .
$$

(7.10) implies that (4.9) holds, i.e., the deformation field at time $t$ is homogeneous. (4.9) and (7.1) now imply that the rotation field $\mathbf{R}(t)$ is also constant. By (7.3) and $(7.9)_{2}, \mathbf{C}^{t}(\tau)$ must also be a constant tensor field. We conclude, at last, that (4.11) must hold; the controllable motions must be homogeneous. It is easy to see now that the results of Section 5 now apply to materials of the triclinic, monoclinic and rhombic systems.

\section{Representations of Response Functionals}

In this section we will summarize the method of constructing representations for response functionals $\mathfrak{T}$ which are form-invariant under the transformations of an arbitrary material symmetry group. The general forms (7.5) and (7.7) will then be verified.

Associated with each symmetry group is a finite set of basic tensors, $\boldsymbol{\alpha}^{(1)}, \ldots, \boldsymbol{\alpha}^{(n)}$, of orders $r(\mathbf{1}), \ldots, r(n)$, respectively, called the anisotropic tensors [10] for that group. Each tensor $\boldsymbol{\alpha}^{(p)}$ has the property that its components are unaltered by the transformations of the symmetry group i. e.,

$$
\boldsymbol{\alpha}_{i_{1} i_{2}}^{(p)} \cdots i_{r(p)}=H_{i_{1} j_{1}} H_{i_{2} j_{2}} \ldots H_{i_{r(p)} j_{r(p)}} \boldsymbol{\alpha}_{j_{1} j_{2}}^{(p)} \cdots j_{r(p)}
$$

for each transformation $\mathbf{H}$ of the symmetry group $\{H\}$. If $\mathbf{H}^{\prime}$ is an orthogonal transformation not in $\{H\}$, then (8.1) fails to hold for at least one of the anisotropic tensors $\boldsymbol{\alpha}^{(1)}, \ldots, \boldsymbol{\alpha}^{(n)}$. For example, for the full isotropy group, the set of anisotropic tensors consists solely of the KRONECKER delta, $\delta_{i j}$.

The primary use of anisotropic tensors is in the construction of certain basic scalar invariants and basic form-invariant tensor-valued functions in terms of which $\mathfrak{T}$ will be expressed. In [11], PIPKIN and RrvLIN illustrated the use of the anisotropic tensors for the special case in which the 
response functionals reduce to polynomials of, say, $\mathbf{B}^{-1}$ and a finite number of vectors. GREEN and RIVLIN [12] used essentially this technique in obtaining the representation for response functionals for isotropic materials which depend on a single second order tensor history $\mathbf{C}^{t}(\tau)$.

The first step in developing the representation for $\mathfrak{z}$ is to construct a set of basic scalar invariants. Each scalar invariant has the form

$$
\begin{aligned}
& I\left[\overline{\mathbf{C}^{t}}\left(\tau_{1}\right), \ldots, \overline{\mathbf{C}}^{t}\left(\tau_{m}\right) ; \overline{\mathbf{B}} ; \boldsymbol{\alpha}^{(1)}, \ldots, \boldsymbol{\alpha}^{(n)}\right]=I\left[\overline{\mathbf{C}}^{t}\left(\tau_{\beta}\right) ; \overline{\mathbf{B}}\right] \\
& \quad=\alpha_{i_{1} j_{1}} \ldots i_{p^{j_{q}}} \ldots i_{i_{r} i_{s}} \bar{C}^{t}{ }_{i_{1} j_{1}}\left(\tau_{1}\right) \ldots \bar{B}_{i_{p^{j}} j_{q}} \ldots \bar{C}^{t} t_{i_{r} i_{s}}\left(\tau_{m}\right),
\end{aligned}
$$

where $\alpha_{i_{1} j_{1}} \ldots i_{r i_{s}}$ is an outer product formed from $\boldsymbol{\alpha}^{(1)}, \ldots, \boldsymbol{\alpha}^{(n)}$. A complete set of basic scalar invariants, denoted by $I$, is constructed by using all possible combinations of $\boldsymbol{\alpha}^{(1)}, \ldots, \boldsymbol{\alpha}^{(n)}$ and $\overline{\mathbf{B}}, \overline{\mathbf{C}}^{t}\left(\tau_{1}\right), \ldots$, $\overline{\mathbf{C}}^{t}\left(\tau_{m}\right)$ in (8.2) and then eliminating those invariants which can be shown to be redundant. Each such invariant is considered a function of $\tau_{1}, \ldots, \tau_{m}$ and satisfies the following invariance condition for each transformation $\mathbf{H}$ of the material symmetry group $\{H\}$,

$$
I\left[\overline{\mathbf{C}}^{t}\left(\tau_{\beta}\right) ; \overline{\mathbf{B}}\right]=I\left[\mathbf{H} \overline{\mathbf{C}}^{t}\left(\tau_{\beta}\right) \mathbf{H}^{T} ; \mathbf{H} \overline{\mathbf{B}} \mathbf{H}^{T}\right] .
$$

In a similar fashion one constructs a set of basic form-invariant tensorvalued functions. Each has the form

$$
\begin{aligned}
& P_{i j^{(\nu)}}\left[\overline{\mathbf{C}}^{t}\left(\tau_{1}\right), \ldots, \overline{\mathbf{C}}^{t}\left(\tau_{m}\right) ; \overline{\mathbf{B}} ; \boldsymbol{\alpha}^{(1)}, \ldots, \boldsymbol{\alpha}^{(n)}\right]=P_{i j}{ }^{(\nu)}\left[\overline{\mathbf{C}}^{t}\left(\tau_{\beta}\right) ; \overline{\mathbf{B}}\right]= \\
& =\alpha_{i j i_{1} j_{1}} \ldots i_{i^{j} j_{q}} \ldots i_{r} i_{s} \bar{C}_{i_{1} j_{1}}\left(\tau_{1}\right) \ldots \bar{B}_{i_{p} j_{q}} \ldots \bar{C}_{i_{r} i_{s}}\left(\tau_{m}\right)
\end{aligned}
$$

where $\alpha_{i j i_{1} j_{1}} \ldots i_{\gamma} i_{s}$ is an outer product of $\boldsymbol{\alpha}^{(1)}, \ldots, \boldsymbol{\alpha}^{(n)}$ in some order. $\mathbf{P}^{(v)}$ is to be considered a function of $\tau_{1}, \ldots, \tau_{m}$. Each tensor-valued function (8.4) satisfies a condition similar to (7.4),

$$
\mathbf{P}^{(v)}\left[\mathbf{H} \overline{\mathbf{C}}^{t}\left(\tau_{\beta}\right) \mathbf{H}^{T} ; \mathbf{H} \overline{\mathbf{B}} \mathbf{H}^{T}\right]=\mathbf{H} \mathbf{P}^{(v)}\left[\overline{\mathbf{C}^{t}}\left(\tau_{\beta}\right) ; \overline{\mathbf{B}}\right] \mathbf{H}^{T} .
$$

For this reason the set formed by eliminating redundancies of form (8.4) is called a set of basic form invariant tensors.

Wineman and Pipkis [13] have shown that any form-invariant response functional can be represented in a certain canonical form in terms of the basic scalar invariants $I$ and the basic form invariant tensors $\mathbf{P}^{(v)}$. Representations of $\mathfrak{i}$ having this form and which are of most use for our present purposes are given by

$$
\begin{aligned}
\mathfrak{T}= & \sum_{v=1}^{N} \phi^{(v)} \mathbf{P}^{(v)}[\overline{\mathbf{B}}]+\int_{t-T}^{t} \theta^{(v+1)}(t-\tau ; I) \mathbf{P}^{(v+1)}\left[\overline{\mathbf{C}}^{t}(\tau) ; \overline{\mathbf{B}}\right] d \tau+ \\
& +\ldots+\underbrace{\iint \ldots \int^{t}}_{t-T} \theta^{(\mu)}\left[t-\tau_{1}, \ldots, t-\tau_{l} ; I\right] \mathbf{P}^{(\mu)} . \\
& \cdot\left[\overline{\mathbf{C}}^{t}(\tau)_{1}, \ldots, \overline{\mathbf{C}}^{t}\left(\tau_{l}\right) ; \overline{\mathbf{B}}\right] d \tau_{1} \ldots d \tau_{l}
\end{aligned}
$$


where tensors $\mathbf{P}^{(v)}, y=1.2, \ldots, N$, are independent of $\overline{\mathbf{C}}^{t}(\tau) . \theta^{(v+1)}$, $\ldots, \theta\left({ }_{\mu}\right)$ are material scalar functions of $\tau_{1}, \ldots, \tau_{l}$. They can also be taken as functions of multiple integrals of the scalar invariants $I$. It is readily verified that (8.6), with (8.3) and (8.5) satisfies (7.4).

The anisotropic tensors for 31 of the 32 crystal classes are derived in [14]. The anisotropic tensors for the remaining class, the gyroidal class of the cubic system, have not been determined.

\section{Cubic System}

For each crystal class of the cubic system, except the gyroidal class, the corresponding set of anisotropic tensors contains the KRONECKER delta, $\delta_{i j}$. The forms of the invariants for a single symmetric tenscr and a vector which have been calculated for the gyroidal class listed in [15] are such that it appears that $\delta_{i j}$ will be one of the anisotropic tensors for the class. We will assume this is so. The remaining anisotropic tensors in the set serve to distingtuish the given crystal class from all the others.

From (8.2) and the fact that $\delta_{i j}$ is an anisotropic tensor, we see that the basic scalar invariants for each crystal class of the cubic system contains as a subset the invariants (2.8), and the kernel $C^{t}{ }_{i j}(\tau)$ of $(2.9)$. Furthermore, from (8.4) we see that among the basic form invariant tensors $\mathbf{P}^{(\prime)}$ are

$$
\mathbf{P}^{(1)}=\mathbf{1}, \mathbf{P}^{(2)}=\overline{\mathbf{B}}, \mathbf{P}^{(3)}=\overline{\mathbf{B}}^{2}, \mathbf{P}^{(n+1)}=\overline{\mathbf{C}}^{t}(\tau) .
$$

By (8.6), (8.7) and the preceding remarks, it follows that possible response functionals for materials of the cubic system have the form (7.5).

Materials of the Tetragonal System, Hexagonal System and Having Transverse Isotropy.

The anisotropic tensors for transversely isotropic materials are [10],

$$
\delta_{3 i}, \alpha_{i j}=\delta_{1 i} \delta_{1 j}+\delta_{2 i} \delta_{2 j} .
$$

The anisotropic tensors for each class of the tetragonal and hexagonal systems contains among them $\alpha_{i j}$ and either $\delta_{3 i}$ or $\delta_{3 i} \delta_{3 j}$. The condition that $\delta_{3 i}$ or $\delta_{3 i} \delta_{3 j}$ satisfy (8.1) restricts the transformations $\mathbf{H}$ of the symmetry group to rotations in a plane and certain reflections. The condition that $\alpha_{i j}$ satisfy (8.1) gives no further restrictions on the transformations. Thus, there is no loss in generality if $\alpha_{i j}$ is replaced by $\alpha_{i j}+\delta_{3 i} \delta_{3 j}=\delta_{i j}$. By the same discussion as was used for the cubic system, we see that possible response functionals the present classes of materials also have the form (7.5).

Triclinic, Monoclinic, and Rhombic Systems

For each crystal class in these systems, the set of anisotropic tensors contains $\alpha_{i}{ }^{(1)}=\delta_{1 i}$ or $\alpha_{i j}{ }^{(1)}=\delta_{1 i} \delta_{1 j}, \alpha_{i}^{(2)}=\delta_{2 i}$ or $\alpha_{i j}{ }^{(22)}=\delta_{2 i} \delta_{2 j}, \alpha_{i}{ }^{\left({ }^{(3)}\right.}=$ $=\delta_{3 i}$ or $\alpha_{i j}{ }^{(33)}=\delta_{3 i} \delta_{3 j}$ and possibly other products of this form. In each case, upon substituting these tensors into (8.2) in all possible combinations, 
it is found that each component $\bar{B}_{i j}, \bar{C}_{i j}{ }^{t}(\tau)$ or the form $\bar{B}_{i j} \bar{B}_{i j}$, $\bar{C}_{p q}\left(\tau_{1}\right) \bar{C}_{p q}\left(\tau_{2}\right)$ (no summation intended) is one of the basic scalar invariants. Similarly, it is found that upon substituting these tenscrs into (8.4) in all possible combinations, the set of basic form-invariant tensors contains, among others,

$$
P^{(1)}=\boldsymbol{\alpha}^{(11)}, P^{(2)}=\boldsymbol{\alpha}^{(22)}, P^{(3)}=\boldsymbol{\alpha}^{(33)} .
$$

Since these form invariant tensors are formed as outer products of anisotropic tensors, they automatically satisfy (8.1). $\alpha^{(22)}$ and $\alpha^{(33)}$ satisfy (8.1) only for orthogonal transformations whose matrices have components $H_{i j}=0, i \neq j$ and $H_{i j}= \pm 1, i=j$. The condition that $\boldsymbol{\alpha}^{(11)}$ must satisfy (8.1) yields no further restrictions on the transformations. Thus, there is no loss in generality if $\mathbf{P}^{(1)}=\alpha^{(11)}$ is replaced by $\mathbf{P}^{(1)}=1$, which satisfies (8.1) for all orthogonal transformations.

In view of these remarks, we see that the response functionals for materials of the triclinic, monoclinic and rhombic systems can always be written in the form (7.7).

\section{Acknowledgment}

The work described in this paper was carried out under a grant from the National Science Foundation, whose support is gratefully acknowledged.

\section{References}

[1] Nols, W.: A Mathematical Theory of the Mechanical Behavior of Continuous Media, Arch. Rational Mech. Anal. 2, 197 (1958).

[2] Coleman, B. D. and C. Trunsdell: Homogeneous Motions of Incompressible Materials. ZAMM, 45, 547 (1965).

[3] Truasdeld, C. and W. Noll: The Non-Linear Field Theories of Mechanics. Flugges Encyclopedia of Physics, Vol. III/3, Berlin: Springer, 1965.

[4] ERICKSEn, J. L.: Deformations Possible in Every Isotropie Incompressible. Perfectly Elastic Body. ZAMP, 5, 466 (1954).

[5] Ericksen, J. L.: Deformations Possible in Every Compressible, Isotropic, Perfectly Elastic Material. J. Math. and Phys. XXIV, 126 (1955).

[6] Rivlrin, R. S.: Non-Linear Viscoelastic Solids. SIAM Rev., 7, 3, 323 (1965).

[7] Courant and HrLbert: Methods of Mathematical Physies. Vol. 1, Inter-science Publishers, Inc., New York, 1953.

[8] Colemax, B. D. and W. Noll: Foundations of Linear Viscoelasticity. Rev. Mod. Phys. 33, 239 (1961).

[9] Halmos, P. R.: Finite Dimensional Vector Spaces. Princeton, New Jersey: D. Van Nostrand Company, Inc., 1958.

[10] Smith, G. F. and R. S. RIvLin: The Anisotropic Tensors. Quart. Appl. Math. 15,308 (1957).

[11] Pipkin, A. C. and R. S. Rivirn: The Formulation of Constitutive Equations in Continuum Physics, I. Arch. Rational Mech. Anal., 4, 129 (1959).

[12] Green, A. E. and R. S. Rivlin: The Mechanics of Non-Linear Materials with Memory, Part X. Arch. Rational Mech. Anal., 1, 1 (1957).

[13] Wineman, A.S. and A.C. Pipkin: Material Symmetry Restrictions on Constitutive Equations. Arch. Rational Mech. Anal., 17, 184 (1964). 
118 A. S. Wineman: Controllable Motions of Compressible Simple Materials

[14] Smith, G. F. and R. S. Rrvurn: Integrity Bases for Veetors. The Crystal Classes. Arch. Rational Mech. Anal., 15, 169 (1964).

[15] Smith, G. F., M. M. SMItH, and R. S. RIVLIN : Integrity Bases for a Symmetric Tensor and a Vector - The Crystal Classes. Arch. Rational Mech. Anal. 12, 93 (1963).

Prolessor Dr. A. S. Wineman

Dept. of Engineering Mechanics

The University of Michigan Ann Arbor, Michigan 48104, U.S.A.

Eigentümer, Herausgeber und Verleger: Springer-Verlag, A-1010 Wien, Mölkerbastei 5. - Für den Textteil verantwortlich: Prof. Dr. H. Parkus, A-1040 Wien, Technische Hochschule, Karisplatz 13. - Für den Anzeigenteil verantwortlich: Alois Hailwax, A-1030 Wien, Paracelsusgasse 8. - Druck: Paul Gerin, A-1021 Wien, Zirkusgasse 13. 